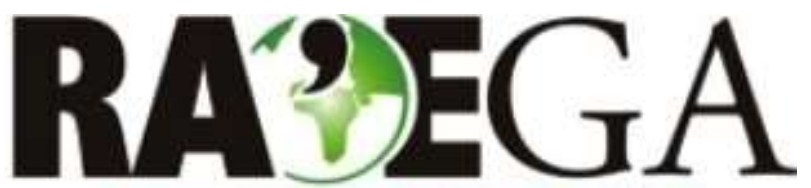

O ESPACYO GEOGRÁFICO EM ANÁLISE

\title{
CONCENTRAÇÃO DIÁRIA DA PRECIPITAÇÃO NA BACIA DO ALTO IGUAÇU, PARANÁ, BRASIL CONCENTRATION INDEX OF RAINFALL IN THE UPPER BASIN IGUAÇU, PARANÁ, BRAZIL
}

\author{
Gabriela Marques Pinheiro ${ }^{1}$, Javier Martin Vide ${ }^{2}$
}

\section{RESUMO}

A Bacia do Alto Iguaçu, localizada na região sul do Brasil, mais precisamente na Região Metropolitana de Curitiba, encontra-se exposta a riscos derivados das inundações, sobretudo nos meses de verão (dezembro, janeiro e fevereiro). Durante este período, a bacia experimenta precipitações convectivas associadas a sistemas frontais juntamente com o aquecimento local e a entrada de umidade procedente do norte do Brasil. Dada a sua concentração e seu grande volume total, o conhecimento da precipitação em escala diária é essencial para o planejamento e gestão da bacia. Em particular, a compreensão dos padrões de precipitação diária torna-se essencial para o planejamento e gestão do território, assim como para a conservação dos recursos naturais. Com base no conhecimento da área de estudo, neste trabalho utilizou-se o índice de concentração da precipitação (Cl) para determinar os potencias impactos da precipitação diária. O índice foi aplicado para 18 estações meteorológicas com dados homogeneizados de qualidade, para o período de 1980 a 2010. Os valores foram calculados considerando os totais e a sazonalidade, com o intuito de verificar possíveis variações. Os valores do índice de concentração, foram altos durante o outono e inverno, já durante a primavera e o verão, apresentaram-se moderados.

PALAVRAS-CHAVE: Bacia do Alto Iguaçu, precipitação, variabilidade e índice de concentração da precipitação.

\section{ABSTRACT}

The Upper Iguaçu Basin/Paraná, located in the South of Brazil, is exposed to flood risk, mainly during summer months (December, January and February). During this period, the basin undergoes convective precipitation associated with frontal systems with local heating. Besides that, the convective precipitation is related to the moisture entrance from the North of Brazil. Given its concentration at time and large total volume, the knowledge of the resolution of daily rainfall is very important. Particularly, the comprehension of the daily rainfall patterns is essential for planning and management of land and to the conservation of natural resources. Based on area knowledge, this paper uses the concentration index (IC) to determine the potential impacts of daily rainfall. The index was applied in 18 meteorological stations within homogenized quality for the period 1970-2010. The values were calculated considering the the total amount and the sazonality, with the purpose of check possible variations.The values of the Concentration Index were high during fall and winter, and during summer and spring it reveals to be moderate.

KEY-WORDS: Iguaçu basin, rainfall, variability and concentration index

\footnotetext{
${ }^{1}$ Universidade Estadual do Centro-Oeste - UNICENTRO Irati, PR, Brasil, e-mail: gabimpinheiro20@hotmail.com

${ }^{2}$ Universitat de Barcelona, Barcelona,Espanha, e-mail: jmartinvide@ub.edu
} 


\section{PINHEIRO,G.M., VIDE,J.M. \\ CONCENTRAÇÃo DIÁRIA DA PRECIPITAÇÃO NA BACIA DO ALTO IGUAÇU, PARANÁ, BRASIL}

\section{INTRODUÇÃO}

Segundo as perspectivas do IPCC (International Panel on Climate Change), as grandes cidades tendem a concentração populacional, gerando graves problemas sociais e ambientais de todas as classes. Enquanto os riscos e vulnerabilidades, perante os problemas ambientais urbanos, tendem a intensificar, os estudos os quais permitam os enfrentamentos, mitigações e adaptações, perante as inundações urbanas, é necessário.

Os eventos climáticos extremos de precipitação estão entre as causas deflagradoras de desastres naturais que afetam a sociedade. Os regimes das chuvas e sua variabilidade natural são afetados pelas transformações da superfície da terra, passando por períodos irregulares, marcados por períodos chuvosos e secos, quentes e frios, responsáveis por inúmeras repercussões espaciais.

Desta forma, os fenômenos associados com as flutuações pluviométricas, como episódios de enchentes e inundações, são constantes, principalmente nas grandes cidades do Sul e Sudeste do Brasil. Os eventos de inundações apresentaram-se críticos entre os anos de 1974 a 2003 e, segundo dados EM-DAT (banco de dados de eventos de emergência) as catástrofes naturais vem aumentando, entre os anos de 1948 e 2007.

De acordo com RAN-1 (Relatório de Avaliação Nacional/Bases Cientificas), do Painel Brasileiro de Mudanças Climáticas (2014), a projeção para mudanças na precipitação para a ampla região Sul da América do Sul prevê, até 2040, aumento de $5 \%$ a $10 \%$ na chuva. Em meados do século (2041-2070), mantêm-se as tendências de aumento gradual de $15 \%$ a $20 \%$ nas chuvas, sendo que essas tendências acentuam-se ainda mais no final do século (2071-
2100), com padrões de clima entre $25 \%$ a $30 \%$ mais chuvoso.

Segundo as hipóteses vinculadas pelo IPCC, a Região Sul do Brasil será afetada futuramente com a ocorrência de cenários de climas mais extremos, com ênfase para o aumento da frequência de eventos extremos ocasionando maior ocorrência de inundações e alagamentos. Além do volume pluviométrico, a vulnerabilidade dos municípios da região contribui para a gravidade do evento.

Dentro deste contexto, a pluviosidade é uma das mais importantes variáveis meteorológicas para os estudos climáticos. Essa importância deve-se a sua função dentro da dinâmica climática e de sua variação no espaço e no tempo. Além disso, ela constitui uma das variáveis climáticas que mais influência no cotidiano das pessoas e na qualidade do meio ambiente. Os volumes de precipitação pluvial, a distribuição temporal e a intensidade das chuvas afetam a sociedade, a economia e a dinâmica natural do planeta.

Desta maneira, o artigo tem como objetivo compreender os padrões da precipitação diária utilizando o índice de concentração $(\mathrm{Cl})$, desenvolvido por Martín-Vide (2004). Este índice é utilizado para compreender as variações das precipitações em diferentes escalas de tempo e sua correlação é importante, pois a concentração da precipitação representa o potencial de ocorrência de inundações. Assim, este artigo, limitou-se ao estado do Paraná, mais precisamente à Bacia do Alto Iguaçu, região metropolitana de Curitiba, Figura 01. 


\section{CONCENTRAÇÃO DIÁRIA DA PRECIPITAÇÃO NA BACIA DO ALTO IGUAÇU, PARANÁ, BRASIL}

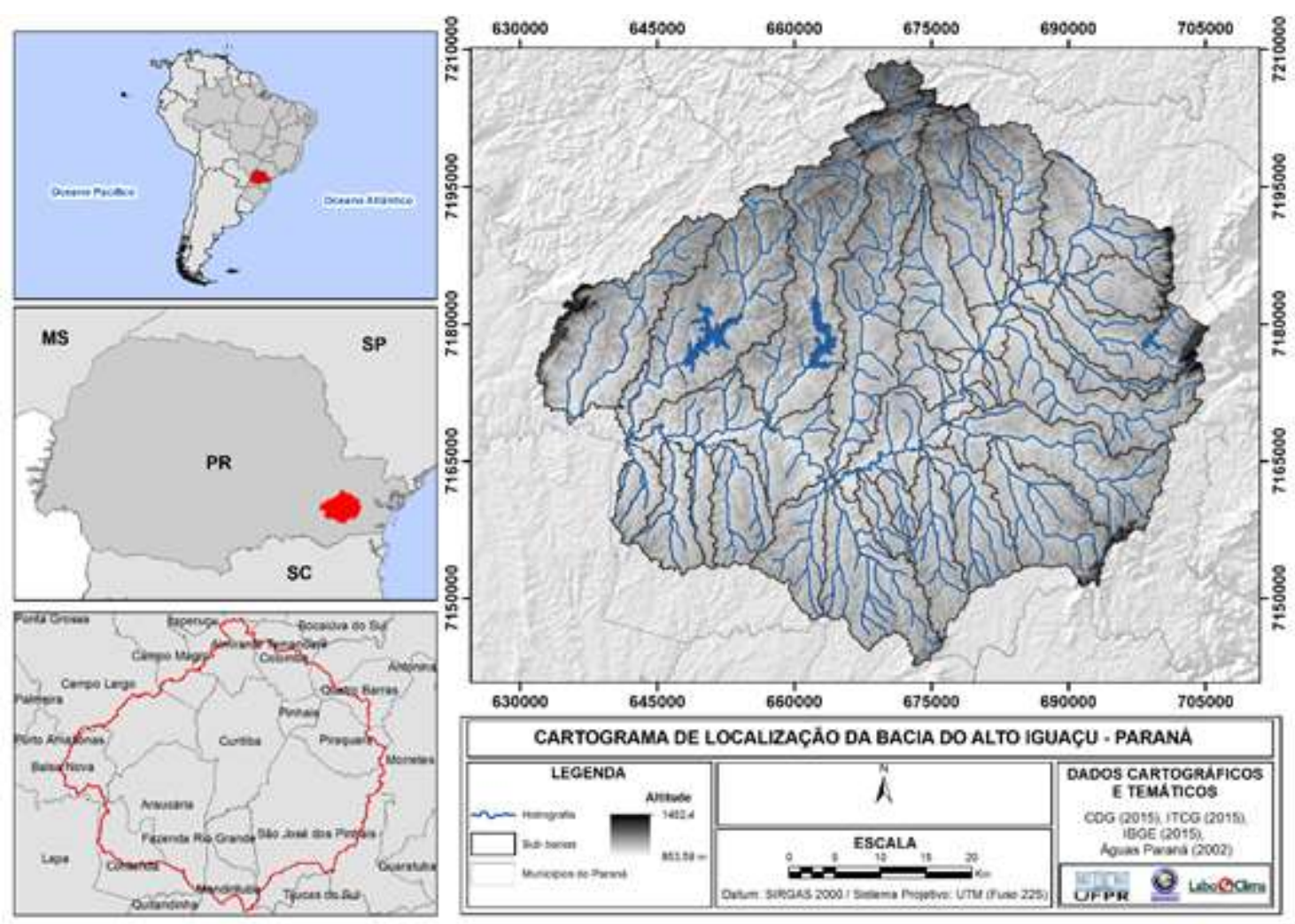

Figura 01 - Localização da Bacia do Alto Iguaçu.

As principais atividades econômicas desenvolvidas na bacia estão relacionadas aos setor A área a ser estudada possui, segundo o IBGE (2010), um total de 2.777.317 habitantes, ocupando uma área total de $3977,7 \mathrm{Km} 2$, com uma densidade média de 983, 75 hab./Km2 e a altitude varia de $850 \mathrm{~m}$ a $1450 \mathrm{~m}$. A região encontra-se inserida no contexto global, o qual constitui parte do cenário das mudanças climáticas globais e participa, portanto, do jogo de causas e consequências do processo. A interação entre a dimensão regional e a dimensão global do fenômeno constitui o centro da análise no que concerne a variabilidade pluviométrica. Dessa forma, o foco principal de análise remete ao estudo da variabilidade pluviométrica e sua repercussão espaçotemporal, associando abordagens qualitativa (dinâmica) e quantitativa (estatística).

A Bacia do Alto Iguaçu foi definida como área de estudo, pois experimentou intenso processo de crescimento da população nas últimas décadas, apresentando tendência de crescimento da população e continuidade na taxa deste crescimento, sendo que a perspectiva é de $2,1 \%$ a.a., entre os anos de 2010 e 2020. As estimativas são de 1,39 milhões de pessoas até 2020 no núcleo central e de 3,5 e 4,2 milhões de habitantes na RMC.

\section{MATERIAIS E MÉTODOS}

$\mathrm{O}$ método de Concentration Index $(\mathrm{Cl}$, sigla em inglês), ou Índice de Concentração, foi idealizado e aperfeiçoado por Martín-Vide (2004). O autor explica que em Climatologia, os dados diários são fundamentais para a qualidade das investigações, uma vez que fornecem referências que admitem conhecer a estrutura temporal fina dos fenômenos, possibilitando a compreensão do comportamento e a concentração da precipitação diária, auxiliando na planificação do território e na gestão e conservação dos recursos naturais.

De acordo com o IPCC (2007), os padrões da precipitação diária estão cada vez mais influenciados pelas situações de mudanças 


\section{CONCENTRAÇÃO DIÁRIA DA PRECIPITAÇÃO NA BACIA DO ALTO IGUAÇU, PARANÁ, BRASIL}

climáticas, consequentemente as situações de vulnerabilidade estão associadas à intensidade e frequência dos eventos climáticos extremos.

A distribuição da frequência das quantidades diárias de precipitação está, em geral, ajustada mediante a distribuição exponencial negativa (BROOKS E CARRUTHERS, 1953). Desta forma, existem mais dias de precipitação com pequenas quantidades do que com quantidades altas. Estas escassas quantidades de precipitação diária podem ter um considerável peso na porcentagem total de chuva de um determinado lugar e também contribuem de forma direta na capacidade hídrica anual do mesmo (MARTíN-VIDE, 2004). Desta forma, ao determinar as classes de precipitação, de acordo com a frequência de cada uma das estações meteorológica, supõe-se uma primeira aproximação da estrutura temporal da precipitação.

De acordo com Martín-Vide (2004), o cálculo do índice de concentração tem por objetivo quantificar as características das estruturas das quantidades acumuladas de precipitação através do número de dias acumulados de chuva. Este índice pode ser considerado como indicador confiável da intensidade e erosividade da precipitação. Estudos similares de Martín-Vide (2004), Sánchez-Lorenzo y Martín-Vide (2006) e Benhamrouche e Martín-Vide $(2011,2012)$ para a Espanha peninsular; Sarricolea et al. (2013) para Chile central; Wang et al. (2013) para a China central, igualmente concluíram que as regiões onde os valores do $\mathrm{Cl}$ resultaram superiores a 0,60 foram classificados como zonas de maior concentração da precipitação diária e que as chuvas ocorreram com mais agressividade. Desta forma, os resultados podem ser analisados de acordo com a tabela 01, abaixo..

Tabela 01 - Distribuição de frequências em $1 \mathrm{~mm}$ de classes. Frequências acumuladas $X$ versus porcentagens correspondentes da precipitação total Y - Estação Curitiba/Curitiba (1980-2010).

\begin{tabular}{|c|c|c|c|c|c|c|c|}
\hline CLASSE & MARCA & $\mathbf{n}_{\mathrm{i}}$ & $\sum n_{i}$ & $\mathbf{P}_{\mathbf{i}}$ & $\sum P_{i}$ & $\sum n_{i}(\%)=X$ & $\sum P_{i}(\%)=Y$ \\
\hline $0,1-0,9$ & 0,5 & 1.720 & 1.720 & 860,0 & 860,0 & 33,8 & 1,9 \\
\hline $1,0-1,9$ & 1,5 & 490 & 2.210 & 735,0 & $1.595,0$ & 43,5 & 3,5 \\
\hline $2,0-2,9$ & 2,5 & 362 & 2.572 & 905,0 & $2.500,0$ & 50,6 & 5,5 \\
\hline $3,0-3,9$ & 3,5 & 231 & 2.803 & 808,5 & $3.308,5$ & 55,1 & 7,3 \\
\hline $4,0-4,9$ & 4,5 & 197 & 3.000 & 886,5 & $4.195,0$ & 59,0 & 9,2 \\
\hline $5,0-5,9$ & 5,5 & 160 & 3.160 & 880,0 & $5.075,0$ & 62,1 & 11,1 \\
\hline $6,0-6,9$ & 6,5 & 140 & 3.300 & 910,0 & $5.985,0$ & 64,9 & 13,1 \\
\hline $7,0-7,9$ & 7,5 & 129 & 3.429 & 967,5 & $6.952,5$ & 67,4 & 15,3 \\
\hline $8,0-8,9$ & 8,5 & 111 & 3.540 & 943,5 & $7.896,0$ & 69,6 & 17,3 \\
\hline $9,0-9,9$ & 9,5 & 117 & 3.657 & $1.111,5$ & $9.007,5$ & 71,9 & 19,8 \\
\hline $10,0-10,9$ & 10,5 & 99 & 3.756 & $1.039,5$ & $10.047,0$ & 73,8 & 22,1 \\
\hline $11,0-11,9$ & 11,5 & 77 & 3.833 & 885,5 & $10.932,5$ & 75,4 & 24,0 \\
\hline $12,0-12,9$ & 12,5 & 65 & 3.898 & 812,5 & $11.745,0$ & 76,6 & 25,8 \\
\hline $13,0-13,9$ & 13,5 & 82 & 3.980 & $1.107,0$ & $12.852,0$ & 78,3 & 28,2 \\
\hline $14,0-14,9$ & 14,5 & 84 & 4.064 & $1.218,0$ & $14.070,0$ & 79,9 & 30,9 \\
\hline $15,0-15,9$ & 15,5 & 62 & 4.126 & 961,0 & $15.031,0$ & 81,1 & 33,0 \\
\hline $16,0-16,9$ & 16,5 & 73 & 4.199 & $1.204,5$ & $16.235,5$ & 82,6 & 35,7 \\
\hline $17,0-17,9$ & 17,5 & 53 & 4.252 & 927,5 & $17.163,0$ & 83,6 & 37,7 \\
\hline $18,0-18,9$ & 18,5 & 56 & 4.308 & $1.036,0$ & $18.199,0$ & 84,7 & 40,0 \\
\hline
\end{tabular}


PINHEIRO,G.M., VIDE,J.M.

CONCENTRAÇÃO DIÁRIA DA PRECIPITAÇÃO NA BACIA DO ALTO IGUAÇU, PARANÁ, BRASIL

\begin{tabular}{|c|c|c|c|c|c|c|c|}
\hline $19,0-19,9$ & 19,5 & 45 & 4.353 & 877,5 & $19.076,5$ & 85,6 & 41,9 \\
\hline $20,0-20,9$ & 20,5 & 44 & 4.397 & 902,0 & $19.978,5$ & 86,5 & 43,9 \\
\hline $21,0-21,9$ & 21,5 & 49 & 4.446 & $1.053,5$ & $21.032,0$ & 87,4 & 46,2 \\
\hline $22,0-22,9$ & 22,5 & 36 & 4.482 & 810,0 & $21.842,0$ & 88,1 & 48,0 \\
\hline $23,0-23,9$ & 23,5 & 26 & 4.508 & 611,0 & $22.453,0$ & 88,6 & 49,3 \\
\hline $24,0-24,9$ & 24,5 & 30 & 4.538 & 735,0 & $23.188,0$ & 89,2 & 50,9 \\
\hline $25,0-25,9$ & 25,5 & 23 & 4.561 & 586,5 & $23.774,5$ & 89,7 & 52,2 \\
\hline $26,0-26,9$ & 26,5 & 37 & 4.598 & 980,5 & $24.755,0$ & 90,4 & 54,4 \\
\hline $27,0-27,9$ & 27,5 & 37 & 4.635 & $1.017,5$ & $25.772,5$ & 91,1 & 56,6 \\
\hline $28,0-28,9$ & 28,5 & 26 & 4.661 & 741,0 & $26.513,5$ & 91,6 & 58,2 \\
\hline $29,0-29,9$ & 29,5 & 25 & 4.686 & 737,5 & $27.251,0$ & 92,1 & 59,8 \\
\hline $30,0-30,9$ & 30,5 & 23 & 4.709 & 701,5 & $27.952,5$ & 92,6 & 61,4 \\
\hline $31,0-31,9$ & 31,5 & 25 & 4.734 & 787,5 & $28.740,0$ & 93,1 & 63,1 \\
\hline $32,0-32,9$ & 32,5 & 17 & 4.751 & 552,5 & $29.292,5$ & 93,4 & 64,3 \\
\hline $33,0-33,9$ & 33,5 & 27 & 4.778 & 904,5 & $30.197,0$ & 93,9 & 66,3 \\
\hline $34,0-34,9$ & 34,5 & 23 & 4.801 & 793,5 & $30.990,5$ & 94,4 & 68,1 \\
\hline $35,0-35,9$ & 35,5 & 20 & 4.821 & 710,0 & $31.700,5$ & 94,8 & 69,6 \\
\hline $36,0-36,9$ & 36,5 & 17 & 4.838 & 620,5 & $32.321,0$ & 95,1 & 71,0 \\
\hline $37,0-37,9$ & 37,5 & 18 & 4.856 & 675,0 & $32.996,0$ & 95,5 & 72,5 \\
\hline $38,0-38,9$ & 38,5 & 16 & 4.872 & 616,0 & $33.612,0$ & 95,8 & 73,8 \\
\hline $39,0-39,9$ & 39,5 & 12 & 4.884 & 474,0 & $34.086,0$ & 96,0 & 74,9 \\
\hline $40,0-40,9$ & 40,5 & 14 & 4.898 & 567,0 & $34.653,0$ & 96,3 & 76,1 \\
\hline $41,0-41,9$ & 41,5 & 22 & 4.920 & 913,0 & $35.566,0$ & 96,7 & 78,1 \\
\hline $42,0-42,9$ & 42,5 & 7 & 4.927 & 297,5 & $35.863,5$ & 96,9 & 78,8 \\
\hline $43,0-43,9$ & 43,5 & 9 & 4.936 & 391,5 & $36.255,0$ & 97,1 & 79,6 \\
\hline $44,0-44,9$ & 44,5 & 8 & 4.944 & 356,0 & $36.611,0$ & 97,2 & 80,4 \\
\hline $45,0-45,9$ & 45,5 & 7 & 4.951 & 318,5 & $36.929,5$ & 97,3 & 81,1 \\
\hline $46,0-46,9$ & 46,5 & 8 & 4.959 & 372,0 & $37.301,5$ & 97,5 & 81,9 \\
\hline $47,0-47,9$ & 47,5 & 5 & 4.964 & 237,5 & $37.539,0$ & 97,6 & 82,4 \\
\hline $48,0-48,9$ & 48,5 & 9 & 4.973 & 436,5 & $37.975,5$ & 97,8 & 83,4 \\
\hline $49,0-49,9$ & 49,5 & 4 & 4.977 & 198,0 & $38.173,5$ & 97,9 & 83,8 \\
\hline $50,0-50,9$ & 50,5 & 8 & 4.985 & 404,0 & $38.577,5$ & 98,0 & 84,7 \\
\hline $51,0-51,9$ & 51,5 & 7 & 4.992 & 360,5 & $38.938,0$ & 98,2 & 85,5 \\
\hline $52,0-52,9$ & 52,5 & 3 & 4.995 & 157,5 & $39.095,5$ & 98,2 & 85,9 \\
\hline $53,0-53,9$ & 53,5 & 3 & 4.998 & 160,5 & $39.256,0$ & 98,3 & 86,2 \\
\hline $54,0-54,9$ & 54,5 & 3 & 5.001 & 163,5 & $39.419,5$ & 98,3 & 86,6 \\
\hline $55,0-55,9$ & 55,5 & 2 & 5.003 & 111,0 & $39.530,5$ & 98,4 & 86,8 \\
\hline $56,0-56,9$ & 56,5 & 4 & 5.007 & 226,0 & $39.756,5$ & 98,4 & 87,3 \\
\hline $57,0-57,9$ & 57,5 & 4 & 5.011 & 230,0 & $39.986,5$ & 98,5 & 87,8 \\
\hline $58,0-58,9$ & 58,5 & 7 & 5.018 & 409,5 & $40.396,0$ & 98,7 & 88,7 \\
\hline $59,0-59,9$ & 59,5 & 4 & 5.022 & 238,0 & $40.634,0$ & 98,7 & 89,2 \\
\hline $60,0-60,9$ & 60,5 & 1 & 5.023 & 60,5 & $40.694,5$ & 98,8 & 89,4 \\
\hline $61,0-61,9$ & 61,5 & 5 & 5.028 & 307,5 & $41.002,0$ & 98,9 & 90,0 \\
\hline $63,0-63,9$ & 63,5 & 4 & 5.032 & 254,0 & $41.256,0$ & 98,9 & 90,6 \\
\hline
\end{tabular}


PINHEIRO,G.M., VIDE,J.M.

\section{CONCENTRAÇÃO DIÁRIA DA PRECIPITAÇÃO NA BACIA DO ALTO IGUAÇU, PARANÁ, BRASIL}

\begin{tabular}{rrrrrrrr}
\hline $64,0-64,9$ & 64,5 & 3 & 5.035 & 193,5 & $41.449,5$ & 99,0 & 91,0 \\
\hline $65,0-65,9$ & 65,5 & 6 & 5.041 & 393,0 & $41.842,5$ & 99,1 & 91,9 \\
\hline $67,0-67,9$ & 67,5 & 5 & 5.046 & 337,5 & $42.180,0$ & 99,2 & 92,6 \\
\hline $68,0-68,9$ & 68,5 & 3 & 5.049 & 205,5 & $42.385,5$ & 99,3 & 93,1 \\
\hline $69,0-69,9$ & 69,5 & 2 & 5.051 & 139,0 & $42.524,5$ & 99,3 & 93,4 \\
\hline $70,0-70,9$ & 70,5 & 1 & 5.052 & 70,5 & $42.595,0$ & 99,3 & 93,5 \\
\hline $71,0-71,9$ & 71,5 & 1 & 5.053 & 71,5 & $42.666,5$ & 99,4 & 93,7 \\
\hline $72,0-72,9$ & 72,5 & 2 & 5.055 & 145,0 & $42.811,5$ & 99,4 & 94,0 \\
\hline $74,0-74,9$ & 74,5 & 4 & 5.059 & 298,0 & $43.109,5$ & 99,5 & 94,7 \\
\hline $75,0-75,9$ & 75,5 & 1 & 5.060 & 75,5 & $43.185,0$ & 99,5 & 94,8 \\
\hline $76,0-76,9$ & 76,5 & 1 & 5.061 & 76,5 & $43.261,5$ & 99,5 & 95,0 \\
\hline $77,0-77,9$ & 77,5 & 2 & 5.063 & 155,0 & $43.416,5$ & 99,5 & 95,4 \\
\hline $78,0-78,9$ & 78,5 & 2 & 5.065 & 157,0 & $43.573,5$ & 99,6 & 95,7 \\
\hline $79,0-79,9$ & 79,5 & 3 & 5.068 & 238,5 & $43.812,0$ & 99,6 & 96,2 \\
\hline $81,0-81,9$ & 81,5 & 3 & 5.071 & 244,5 & $44.056,5$ & 99,7 & 96,8 \\
\hline $82,0-82,9$ & 82,5 & 1 & 5.072 & 82,5 & $44.139,0$ & 99,7 & 96,9 \\
\hline $83,0-83,9$ & 83,5 & 2 & 5.074 & 167,0 & $44.306,0$ & 99,8 & 97,3 \\
\hline $84,0-84,9$ & 84,5 & 2 & 5.076 & 169,0 & $44.475,0$ & 99,8 & 97,7 \\
\hline $93,0-93,9$ & 93,5 & 2 & 5.078 & 187,0 & $44.662,0$ & 99,8 & 98,1 \\
\hline $95,0-95,9$ & 95,5 & 1 & 5.079 & 95,5 & $44.757,5$ & 99,9 & 98,3 \\
\hline $97,0-97,9$ & 97,5 & 1 & 5.080 & 97,5 & $44.855,0$ & 99,9 & 98,5 \\
\hline $100,0-100,9$ & 100,5 & 2 & 5.082 & 201,0 & $45.056,0$ & 99,9 & 99,0 \\
\hline $106,0-106,9$ & 106,5 & 1 & 5.084 & 106,5 & $45.265,0$ & 100,0 & 99,4 \\
\hline $121,0-121,9$ & 121,5 & 1 & 5.085 & 121,5 & $45.386,5$ & 100,0 & 99,7 \\
\hline $146,0-146,9$ & 146,5 & 1 & 5.086 & 146,5 & $45.533,0$ & 100,0 & 100,0 \\
\hline Totais & & 5.086 & & $45.533,0$ & & $8.061,1$ & $6.068,2$ \\
\hline
\end{tabular}

Ainda sobre esta mesma tabela, nota-se que $71,9 \%$ dos dias de chuvas correspondem apenas a $19,8 \%$ do volume precipitado. Da mesma forma, pode-se assegurar que três quartos (76\%) do volume total das chuvas aconteceram em pouco menos de um quarto $(24,6 \%)$ do total dos dias chuvosos. Para a estação utilizada como modelo, o $\mathrm{Cl}$ resultou em 0,62 , constatando estatisticamente uma alta concentração das chuvas para este posto pluviométrico em relação ao período analisado.

Os resultados anteriores podem ser representados graficamente, por meio das porcentagens dos números acumulados de dias de precipitação em relação ao total de dias de precipitação, que corresponde à penúltima

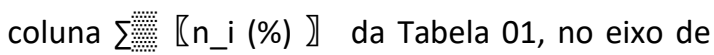
abscissas " $X$ ". Já no eixo de ordenadas, " $Y$ " versus, as porcentagens das quantidades acumuladas dos dias de precipitação com respeito à quantidade total $\sum_{\text {E.w. }}\left[P_{-} i(\%) \rrbracket\right.$, que se refere à última coluna da mesma tabela anteriormente citada. Através do gráfico, é possível verificar que a poligonal resultante tem um aspecto marcadamente exponencial, denominada como curva de concentração de Lorenz. A Figura 02 representa a reta de equidistribuição ou regularidade perfeita da precipitação. A reta representa a igualdade da quantidade de precipitação diária e a curva representa a concentração em função da distância relativa, com referência à reta de equidistribuição. $\mathrm{O}$ autor explica que a poligonal criada debaixo da reta equidistante pode representar irregularidade, deste modo, quanto maior é o distanciamento maior é a concentração e a irregularidade da precipitação, pois quando se 


\section{CONCENTRAÇÃO DIÁRIA DA PRECIPITAÇÃO NA BACIA DO ALTO IGUAÇU, PARANÁ, BRASIL}

tem uma maior área, teoricamente se tem maior anomalia.

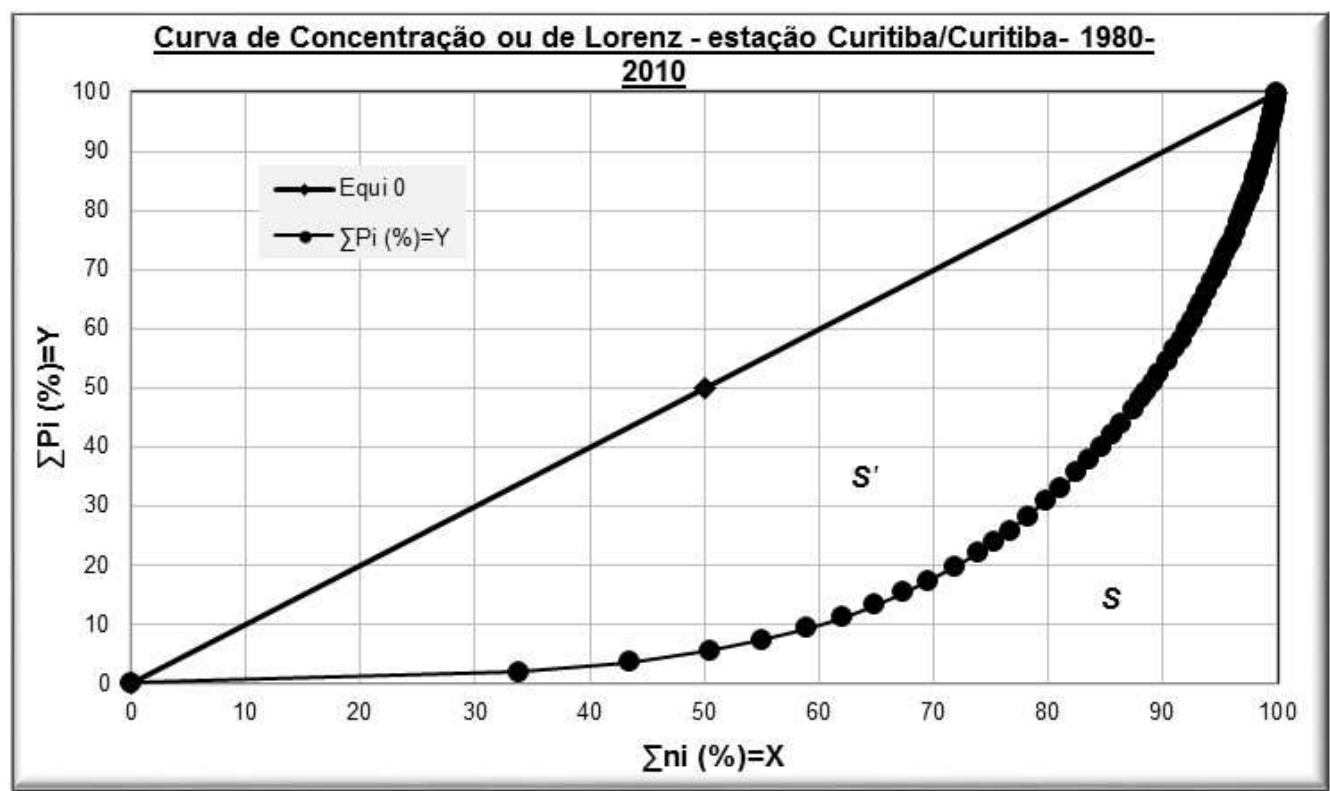

Figura 02 - Curva de Lorenz - Estação de Curitiba/Curitiba (1980-2010).

O índice de concentração de Gini serve para caracterizar e quantificar:

$$
\text { Î́ndice de Gini }=\frac{2 S}{10.000}
$$

O estudo de Martín-Vide (2003) aperfeiçoou o índice anterior mediante a substituição das poligonais pelas curvas exponenciais, posições mais bem ajustadas:

$$
y=a \cdot \bar{x} \cdot a^{b x}
$$

Onde a y b são duas variáveis constantes e podem ser determinadas de acordo com o procedimento de mínimos quadrados e se determinam pelas funções:

$\ln a=\frac{\sum X_{i}^{z} \sum \ln Y_{t}+\sum X_{i} \sum X_{i} \ln X_{i}-\sum X_{i}^{2} \sum \ln X_{i}-\sum X_{i} \sum X_{i} \ln Y_{i}}{N \sum x_{i}^{2}-\left(\sum x_{i}\right)^{2}}$
$\mathbf{b}=\frac{N \sum X_{i} \ln Y_{t}+\sum X_{i} \sum \ln X_{i}-N \sum X_{i} \ln X_{t}-\sum X_{i} \sum \ln Y_{t}}{N \sum x_{i}^{2}-\left(\sum x_{i}\right)^{2}}$
Uma vez determinadas as duas constantes, a integral é definida pela curva exponencial entre 0 e 100 da superfície compreendida entre a curva, eixo das abscissas e a ordenada 100, sendo definida pela aplicação da equação integral abaixo:

$$
S=\left[\frac{a}{b} e^{b x}\left(x-\frac{1}{b}\right)\right]_{0}^{100}
$$

Assim, o índice de concentração da precipitação diária é similar ao de Gini, definido por:

$$
C I=\frac{2 S^{\prime}}{10.000}
$$

A irregularidade ou concentração pluviométrica diária foi avaliada através da separação entre a curva exponencial e a reta de equidistribuição. $\mathrm{O}$ valor de $\mathrm{Cl}$ pode ser valorado pela divisão dos valores ordenados de $S^{\prime}$ pelo valor da área do triângulo (5000), que está localizado abaixo da reta de equidistribuição. Portanto, o valor de $\mathrm{Cl}$ é a fração de $\mathrm{S}^{\prime}$ sobre o valor do triângulo delimitado por uma reta de equidistribuição (MARTíN-VIDE, 2004). O índice de concentração da precipitação diária pode ser definido como:

$$
C I=\frac{S^{\prime}}{5.000}
$$




\section{PINHEIRO,G.M., VIDE,J.M. \\ CONCENTRAÇÃO DIÁRIA DA PRECIPITAÇÃO NA BACIA DO ALTO IGUAÇU, PARANÁ, BRASIL}

Para a realização dos cálculos do índice de concentração da precipitação (Cl) foram utilizados 18 estações pluviométricas, sendo que estes dados foram extraídos da hidrowebsistemas de informação hidrológicas (ANA) e foram homogeneizados através do Programa $R$, pacote Climatol. Já para a realização do calculo do Índice de Concentração da Precipitação foi utilizado o programa R e o pacote Precindicon.

\section{RESULTADOS E DISCUSSÃO}

Entender o comportamento e a concentração da precipitação diária é de grande interesse para a planificação do território e para a gestão e conservação dos recursos naturais. Dessa forma, os padrões de precipitação diário estão cada vez mais influenciados pelo aquecimento global, cada vez mais aceito pela comunidade cientifica, a qual considera que os eventos climáticos extremos, provavelmente ocorreram mais fom frequência. Consequentemente, o nível de agressão da precipitação sobre o solo em meio natural, está diretamente relacionada à intensidade e à distribuição temporal das chuvas. Assim, a intensidade e a quantidade de precipitação possuem uma relação direta sobre a erosão do solo, ocasionando maior vulnerabilidade, produzindo alterações nas estratégias do uso, entre outras coisas (SCHOLZ, 2008).

Sendo assim, a análise da irregularidade e da concentração da precipitação na resolução diária podem ser o primeiro passo para conhecer a estrutura da precipitação em um escala temporal fina. Dessa forma, os resultados são apresentados com a finalidade quantificar as características da estrutura das quantidades acumuladas de precipitação, contribuído pelo número de días acumulados de chuva, mediante o cálculo do índice de concentração (CI) (MARTíN-VIDE, 2004).

$\mathrm{Na}$ Figura 03, estão representados os valores do índice de concentração da precipitação $(\mathrm{Cl})$, para os 31 anos de análise. Os valores abaixo de 0,50 podem ser considerados de concentração baixa da precipitação, entre 0,50 e 0,60 moderados, entre 0,60 a 0,70 altos, e acima de 0,70 muito altos. Nota-se que para todo o período, o maior valor encontrado foi no município de Curitiba, com 0,65, porém outras duas estações apresentaram índices altos, como o município de Colombo e Porto Amazonas, representando $22,2 \%$ do total. Já a maioria das estações apresentaram valores entre 0,50 a 0,60, valores considerados moderados. Estas estações representaram $77,8 \%$ do total analisado.

Analisando a Figura 03, nota-se que as maiores concentrações da precipitação diária encontram-se ao norte da Bacia, mais precisamente nos municípios de Curitiba, Colombo e Campina Grande do Sul, oscilando entre 0,59 a 0,65 . Já os menores valores encontram-se ao noroeste da Bacia, mais precisamente nos municípios de Campo Largo, registrando 0,53 de concentração. Os outros municípios encontram-se com distribuição moderada da concentração diária da precipitação, entre 0,54 a 0,58 .. 


\section{CONCENTRAÇÃO DIÁRIA DA PRECIPITAÇÃO NA BACIA DO ALTO IGUAÇU, PARANÁ, BRASIL}

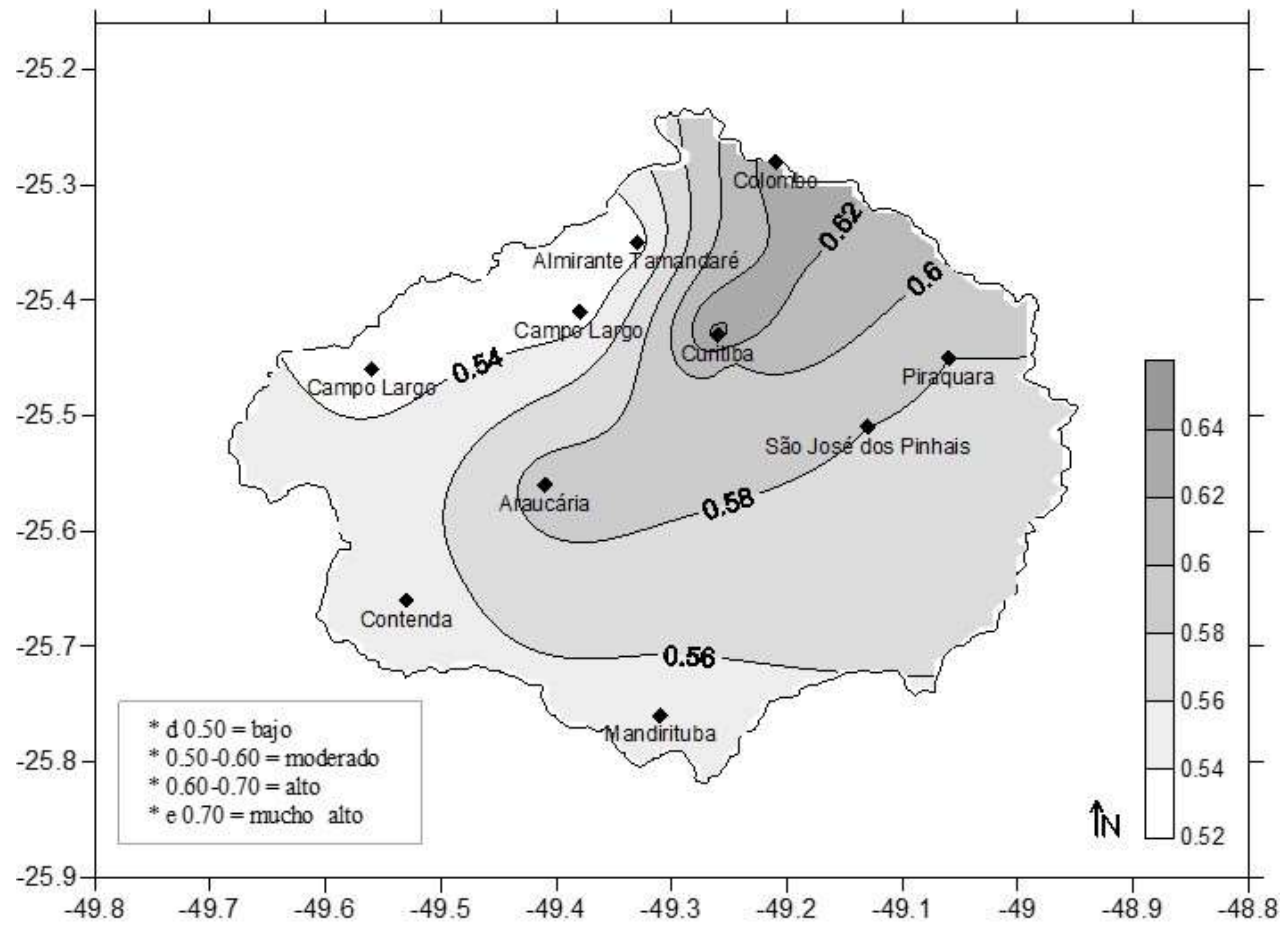

Figura 03 - Bacia do Alto rio Iguaçu/PR - Índice de concentração da precipitação (1980 a 2010).

Analisando sazonalmente a Concentração da Precipitação, nota-se na Figura 04, que durante a primavera, apenas as cidades de Colombo e Curitiba apresentaram concentração acima de 0,60 , considerado alto, segunda a escala de análise. O município de Campo Largo apresentou o menor índice, registrando 0,54. Já a maioria dos municípios manteve o índice entre 0,54 e 0,58, que perante a classificação, é considerada concentração moderada.

Analisando a Figura 04, nota-se que a concentração da precipitação para a primavera está localizada ao norte da Bacia, mais precisamente nos municípios de Curitiba e Colombo, e a menor concentração está localizada ao noroeste da Bacia, no município de Campo Largo. Nota-se, na Figura 03, que este município também apresentou os menores valores para a análise dos totais, assim como o norte apresentou os maiores valores. Diferentemente da análise dos totais, o sul da Bacia apresentouse mais homogêneo, com valores que oscilam entre 0,54 a 0,55. Dessa forma, a análise total e a análise sazonal (primavera) não apresentou muita discrepância espacial. 


\section{CONCENTRAÇÃO DIÁRIA DA PRECIPITAÇÃO NA BACIA DO ALTO IGUAÇU, PARANÁ, BRASIL}

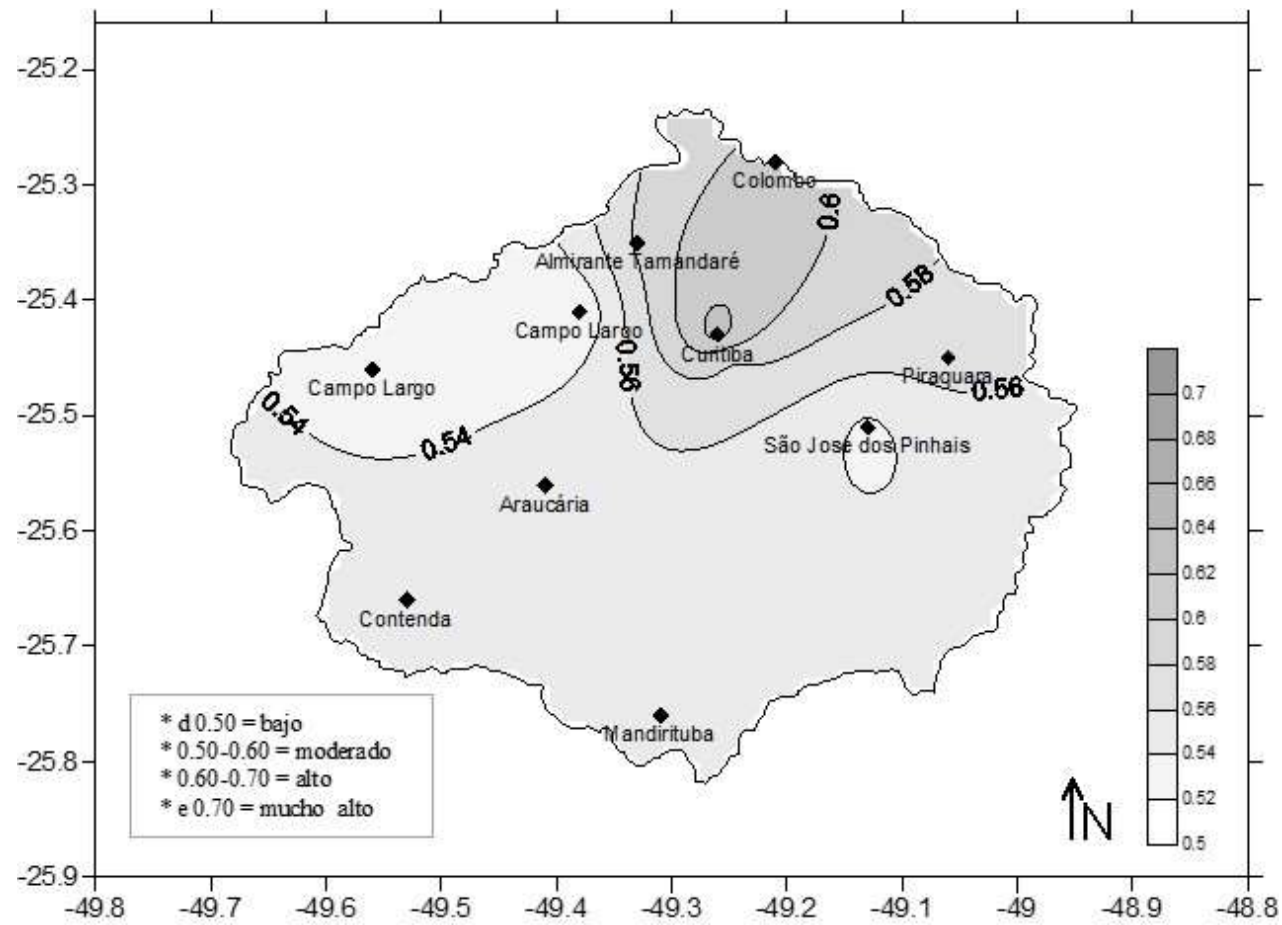

Figura 04 - Bacia do Alto rio Iguaçu/PR - Índice de concentração da precipitação para a primavera (1980 a 2010)

Analisando a Figura 05, observa-se que a concentração da precipitação para os meses de verão oscilaram entre 0,53 a 0,61 , diferentemente da primavera, onde os dados oscilaram entre 0,52 a 0,63. Novamente os municípios de Curitiba e Colombo apresentaram índices acima de 0,60, sendo considerados altos. Os municípios de Almirante Tamandaré, Piraquara e Porto Amazonas registraram, respectivamente, 0,59, 0,58 e 0,58, apresentando índices próximos a 0,60. Novamente os menores índices foram observados no município de Campo Largo, registrando 0,54.

Realizando uma comparação com os dados da primavera, nota-se que o verão apresentou maior concentração da precipitação, como exemplo, podemos citar os municípios de Campina Grande do Sul, Campo Largo e São José dos Pinhais, registrando respectivamente para a primavera, 0,54, 0,52 e 0,55 , e para o verão, $0,56,0,55$ e 0,58 . Porém alguns municípios apresentaram valores semelhantes de concentração, como Colombo, Contenda, Curitiba e Mandirituba.

Analisando a Figura 05, nota-se que a porção norte da Bacia, onde estão localizados os municípios de Curitiba e Colombo, apresentam as maiores concentrações da precipitação e a porção noroeste, as menores concentrações, igualmente para a Figura 03 e Figura 04. 


\section{CONCENTRAÇÃO DIÁRIA DA PRECIPITAÇÃO NA BACIA DO ALTO IGUAÇU, PARANÁ, BRASIL}

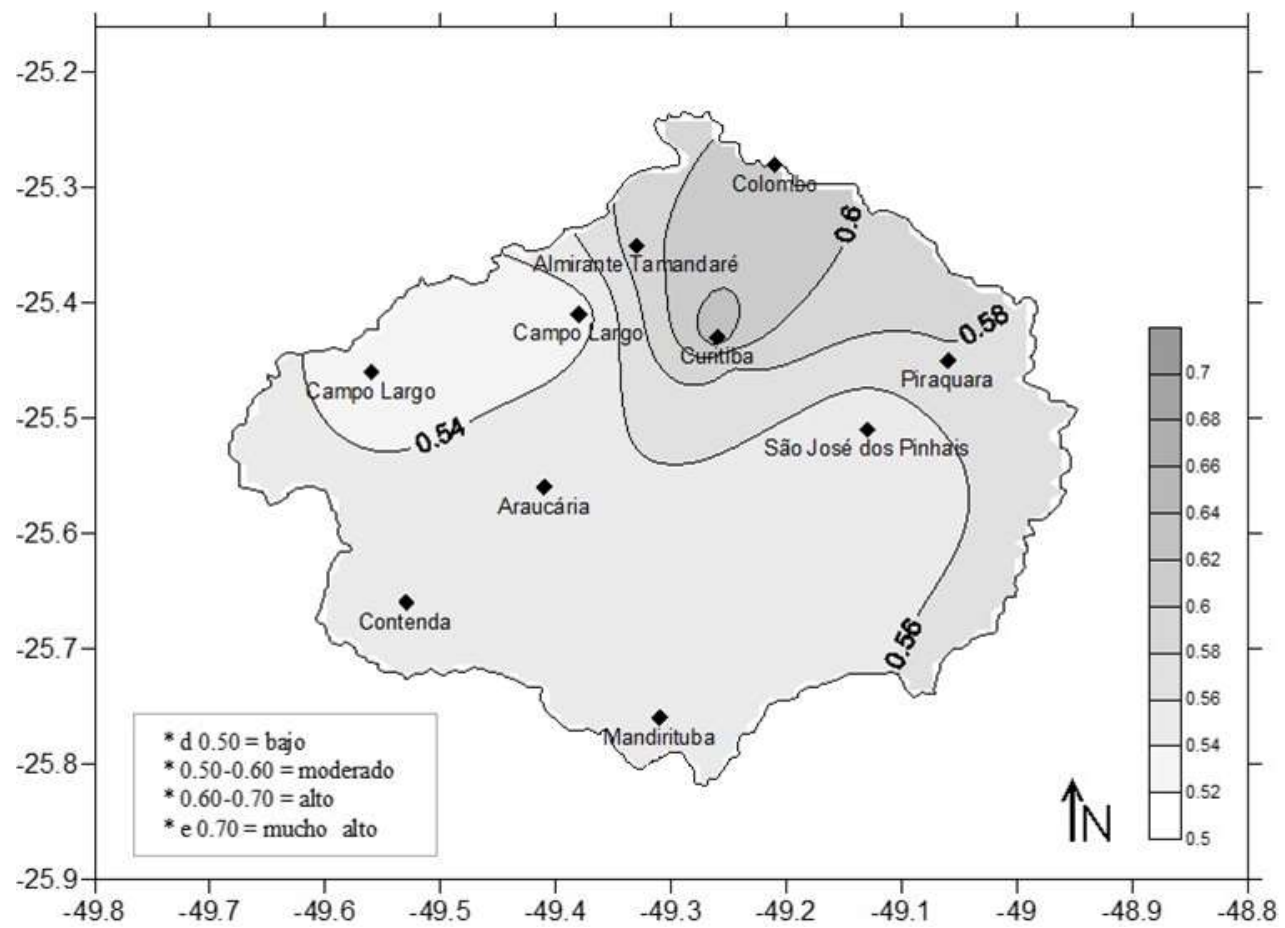

Figura 05 - Bacia do Alto rio Iguaçu/PR - Índice de concentração da precipitação para o verão (1980 a 2010)

Analisando a Figura 06, nota-se que a concentração da precipitação para os meses do outono oscila entre 0,54 e 0,69, diferentemente da primavera e o verão, onde os dados oscilaram entre 0,53 a 0,61 e 0,52 a 0,63. Dessa forma, nota-se que, para o outono, os valores encontram-se mais alto praticamente para todas as estações analisadas, sendo assim, a precipitação apresenta-se mais concentrada nestes meses.

Os municípios de Almirante Tamandaré, Colombo, Curitiba, Piraquara e Porto Amazonas apresentaram índices acima de 0,60, sendo considerados de concentração alta. A estação de Curitiba apresentou o maior valor da série histórica analisada, com 0,69, sendo índices acima de 0,70 , considerados muito alto.

Já a maioria das estações apresentaram índices moderados, estando entre 0,54 e 0,59. 0 município de Campo Largo apresentou o menor índice, registrando 0,54.
Observando a Figura 06, nota-se que a precipitação encontra-se concentrada na porção norte da Bacia, mais precisamente nos municípios de Piraquara, Curitiba, Almirante Tamandaré e Colombo, variando entre 0,60 a 0,69 . Realizando uma comparação com as outras estações do ano, nota-se que a dinâmica assemelha-se, porém o outono apresenta maiores valores.

Novamente, a porção noroeste apresentou os menores valores, oscilando entre 0,54 a 0,56 no município de Campo Largo. O município de Mandirituba apresentou índice de concentração baixo em comparação às outras estações analisadas, registrando 0,54 de concentração. 


\section{CONCENTRAÇÃO DIÁRIA DA PRECIPITAÇÃO NA BACIA DO ALTO IGUAÇU, PARANÁ, BRASIL}

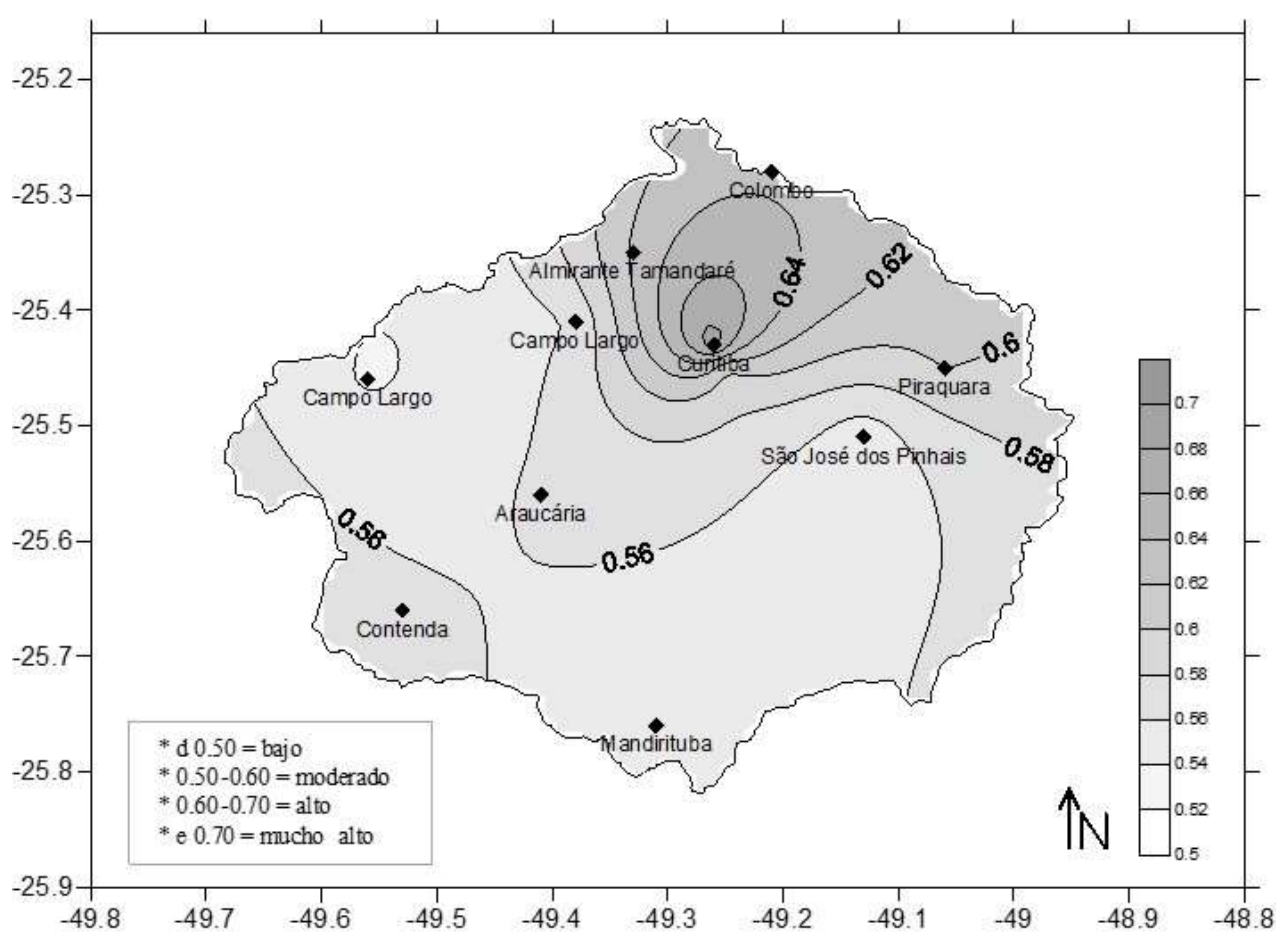

Figura 06 - Bacia do Alto rio Iguaçu/PR - Índice de concentração da precipitação para o outono (1980 a 2010)

Observando a Figura 07, nota-se que os valores da concentração da precipitação para o inverno encontram-se registrados entre 0,53 e 0,67, sendo que, para o outono, os registros oscilaram entre 0,54 e 0,69; para a primavera entre 0,53 e 0,61 ; e para o verão entre 0,52 e 0,63. Os municípios de Almirante Tamandaré, Curitiba, Piraquara e Porto Amazonas apresentaram índices acima de 0,60, sendo considerados altos. Já a maioria das estações apresentaram valores moderados, entre 0,53 a 0,59.

Novamente o município de Campo Largo apresentou o menor índice, registrando 0,55 . Realizando uma comparação com os outros meses de análise, o inverno apresentou a segunda maior concentração da precipitação, estando atrás somente do outono, onde os registros apresentaram-se superiores. Apenas as estações de Mandirituba e São José dos Pinhais, apresentaram valores superiores durante o inverno, em comparação com o outono.

Analisando espacialmente os dados de concentração da precipitação para o inverno, Figura 07 , nota-se que a dinâmica apresentouse semelhante às outras estações do ano, sendo o que as diferenciam são os valores apresentados. Durante o inverno, novamente, o norte da Bacia apresenta os maiores valores, mais precisamente os municípios de Curitiba, Almirante Tamandaré e Colombo. A porção centro-oeste apresentou os menores valores, como os municípios de Araucária, São José dos Pinhais/Fazendinha e Campo Largo.. 


\section{CONCENTRAÇÃO DIÁRIA DA PRECIPITAÇÃO NA BACIA DO ALTO IGUAÇU, PARANÁ, BRASIL}

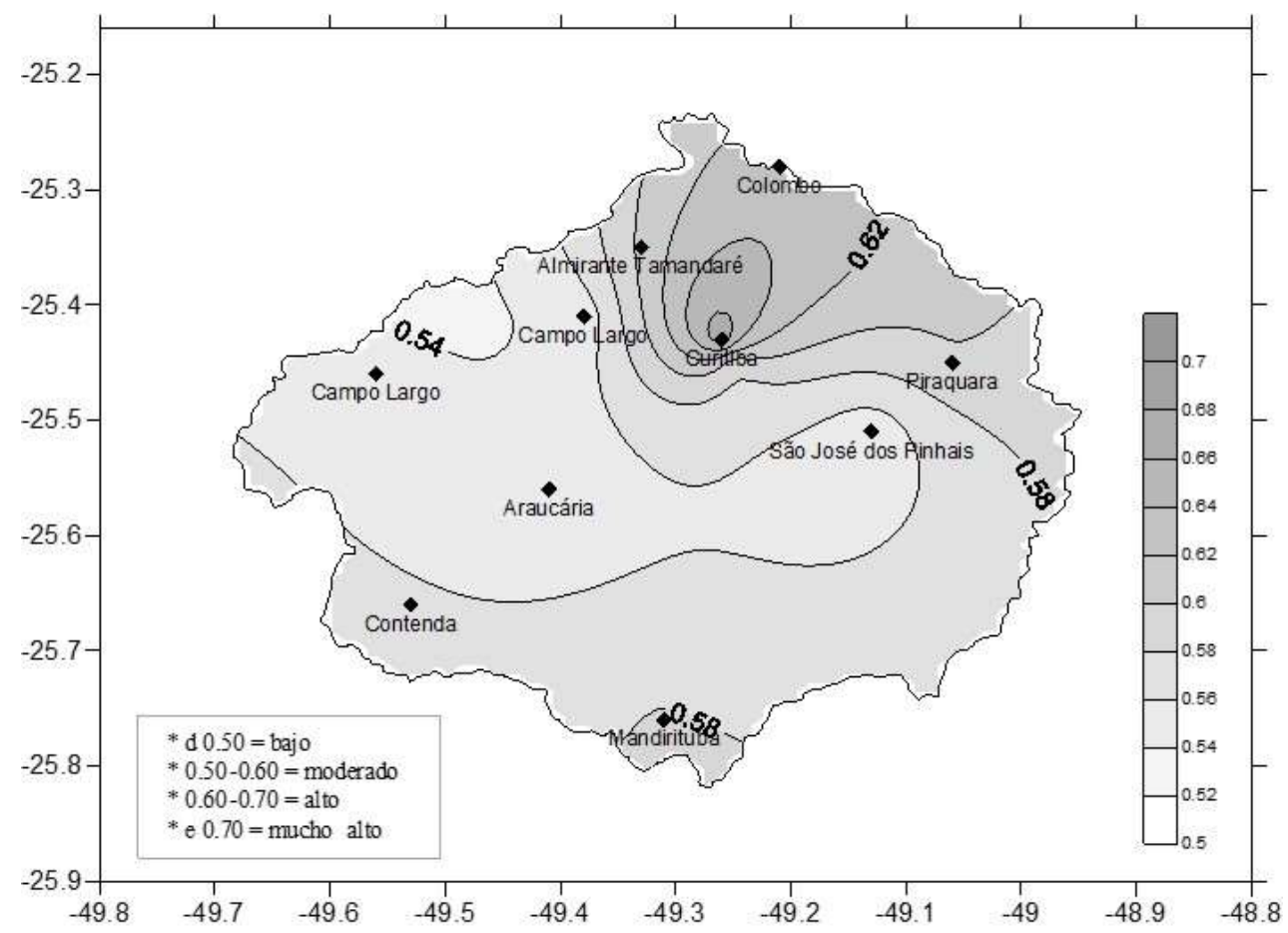

Figura 07. Bacia do Alto rio Iguaçu/PR - Índice de concentração da precipitação para o inverno (1980 a

2010)

\section{CONCLUSÕES}

Em relação à concentração da precipitação, nota-se que o município de Curitiba apresentou a maior concentração da precipitação para os 31 anos de análise, seguido pelo município de Colombo. Na análise sazonal da concentração da precipitação, a dinâmica espacial demostrou-se semelhante para ambas as estações do ano: a porção norte registrou os maiores valores e a porção noroeste apresentou os menores índices. As maiores diferenças foram encontradas em relação aos valores dos índices apresentados para cada uma das estações do ano. O outono e o inverno registraram os maiores valores, índices considerados altos. Já a primavera e o verão apresentaram índices moderados. Dessa forma, pode-se concluir que a concentração da precipitação apresentou-se maior durante o outono e inverno, configurando assim um cenário de chuvas menos intensas, porém constantes.

\section{REFERÊNCIAS}

BENHAMROUCHE, A.; y MARTÍN-VIDE, J.: Avances metodológicos en el análisis de la concentración diaria de la precipitación en la España peninsular. Anales de Geografía, vol. 32, n. 1, p. 11-27, 2012. Disponível em: <http://revistas.ucm.es/index.php/AGUC/article/ view/39306/37879>. Acesso em: 18 jul. 2012.

BENHAMROUCHE, A.; y MARTÍN-VIDE, J.: Distribución espacial de la concentración diaria de la precipitación en la provincia de Alicante. Instituto Interuniversitario de Geografía de la Universidad de Alicante. Investigaciones Geográficas, n. 56, p. 113-129, 2011. Disponível em:

<http://rua.ua.es/dspace/bitstream/10045/2428 5/1/Investigaciones_Geograficas_56_06.pdf >. Acesso em: 02 fev. 2014.

BROOKS, C. E. P. \& CARRUTHERS, N.: Handbook of Statistical Methods in Meteorology. Londres: H. M. Stationery Office, 1953, 412 p.

IPCC, 2007: Climate Change 2007: The Physical Science Basis. Contribution of Working Group I to the Fourth Assessment Report of the Intergovernmental Panel on Climate Change [Solomon, S., D. Qin, M. Manning, Z. Chen, M. Marquis, K.B. Averyt, M.Tignor and H.L. Miller 


\section{CONCENTRAÇÃO DIÁRIA DA PRECIPITAÇÃO NA BACIA DO ALTO IGUAÇU, PARANÁ, BRASIL}

(eds.)]. Cambridge University Press, Cambridge, United Kingdom and New York, NY, USA.

MARTÍN-VIDE, J.: El tiempo y el Clima. Colección Cuadernos de Medio Ambiente. Barcelona: Rubes Editorial, 2003, 128 p.

MARTÍN-VIDE, J.: Spatial distribution of daily precipitation concentration index in Peninsular Spain. International Journal of Climatology, vol. 24, Issue 8, 959-971 p., 2004. Disponível em: <http://onlinelibrary.wiley.com.sire.ub.edu/doi/1 0. 1002/joc.1030/epdf>. Acesso em: 05 mai. 2012.

SARRICOLEA, P.; OSSANDÓN, M. J. H.; y ESCOBAR, C. A.: Análisis de la concentración diaria de las precipitaciones en Chile central y su relación con la componente zonal (subtropicalidad) y meridiana (orográfica). Investig. Geogr. Chile, 45: 37-50 p., 2013. Disponível em: <http://www.revistas.uchile.cl/index.php/IG/ article/viewFile/27595/29261>. Acesso em: 27 mai. 2015.
SÁNCHEZ-LORENZO, A. y MARTÍN-VIDE, J.: Distribución espacial de la concentración pluviométrica diaria en la Península Ibérica. Proceedings 5. Asamblea Hispano-Portuguesa de Geodesia y Geofísica. Sesión Climatología y Cambio Climático. Sevilla, 2006.

SCHOLZ, G.; QUINTON, J. N.; STRAUSS, P. Soil erosion from sugar beet in Central Europe in response to climate change induced seasonal precipitation variations. Catena 72: 2008 p.91105

WANG, W.; XING, W.; YANG, T.; SHAO, Q.; PENG, S.; Yu, Z.; and YONG, B.: Characterizing the changing behaviours of precipitation concentration in the Yangtze River Basin, China. Hydrological Processes, vol. 27, Issue 24, pages: 3375-3393, 2013. Disponível em: <http://onlinelibrary.wiley.com.sire.ub.edu/doi/ 10.1002/hyp.9430/epdf>. Acesso em: 27 mar. 2015. 